\title{
Bullous pemphigoid and neurodegenerative diseases: a study in a setting of a Central European university dermatology department
}

\author{
Paweł Pietkiewicz $^{1} \cdot$ Justyna Gornowicz-Porowska $^{1} \cdot$ Monika Bowszyc-Dmochowska $^{2}$. \\ Paweł Bartkiewicz $^{1} \cdot$ Marian Dmochowski $^{1}$
}

Received: 15 July 2015/Accepted: 18 September 2015/Published online: 29 September 2015

(C) The Author(s) 2015. This article is published with open access at Springerlink.com

\begin{abstract}
Bullous pemphigoid (BP) is an autoimmune blistering dermatosis of the elderly mediated by $\operatorname{IgG}$ and IgE antibodies to skin hemidesmosomal proteins, BP180 and/or BP230, that occur physiologically also in neuronal tissue. It was reported that $\mathrm{BP}$ is associated with neurodegenerative diseases (ND). We performed a retrospective study in a setting of a Central European university dermatology department on prevalence of ND in $94 \mathrm{BP}$ patients. 26 out of $94 \mathrm{BP}$ patients had at least one ND. ND included: Parkinson's disease, dementia, stroke, hear loss, tinnitus, blindness, vertigo, neurosyphilis, systemic sclerosis, and epilepsy. Since population aging is conceivably responsible for the rising number of $\mathrm{BP}$ cases as a result of immunosenescence-related phenomena, the plausible BPspecific immunopathogenetic relationship between BP and ND deserves to be further experimentally explored.
\end{abstract}

Keywords Bullous pemphigoid $\cdot$ Neurodegenerative diseases $\cdot$ Aging

Paweł Pietkiewicz

p.pietkiewicz@mp.pl

1 Autoimmune Blistering Dermatoses Section, Department of Dermatology, Poznan University of Medical Sciences, 49 Przybyszewski Street, 60-355 Poznan, Poland

2 Cutaneous Histopathology and Immunopathology Section, Department of Dermatology, Poznan University of Medical Sciences, Poznan, Poland

\section{Introduction}

Bullous pemphigoid (BP) is an autoimmune blistering dermatosis common among elderly octogenarians and older. IgG- and IgE-mediated autoimmunity to hemidesmosomal proteins, BP180 and/or BP230, and mixed neutrophil/eosinophil infiltrate clinically results in forming tense-top subepidermal blisters on urticariform plaques, erythematous or noninflamed skin, predominantly on the flexural aspects of limps and on the trunk [1-3]. The neuronal forms of these proteins, BP180 (type XVII collagen, BPAG2) and BP230 (dystonin, BPAG1n, BPAG1a), occur physiologically in neuronal tissue and are fundamental for retainment of neuro-cytoskeleton organization. Transmembrane protein BP180 seems to be associated with synapse stabilization in central nervous system and synaptic plasticity of the brain, whereas intracellular protein BP230 anchors the neural intermediate filaments to the actin cytoskeleton, microtubule and microfilament cytoskeletal networks with each other and to distinct cell membrane sites, acting also as a scaffold for signaling proteins that regulate the cytoskeleton alteration [4].

Dystonin (DST) gives many transcript variants of BPAG1 plakin family proteins (due to alternative splicing phenomenon), whereas their role is mostly unclear. There are three main isoforms of BPAG1: BPAG1e (BP230) in stratified epithelia, BPAG1a (or BPAG1n) in neuronal tissue and BPAG1b in striated muscle tissue. Inactivation of DST results in neurodegeneration and blistering in mice and hereditary autonomic sensory neuropathy in human [57]. It was reported that BP is associated with, and in that cases is usually preceded by, neurodegenerative diseases (ND) (e.g. Parkinson's disease, dementia, stroke, epilepsy, multiple sclerosis or even neurosyphilis) [8-11]. It was estimated in a British study that in about $72 \%$ BP patients 
ND preceded the disease by a mean of 5.5 years [12]. Interestingly, it was recently shown that dementia and Parkinson's disease patients without BP may also develop cerebrospinal fluid (CSF) anti-BP180 antibodies to nonpathogenic intracellular and extracellular domains and, rarely, to pathogenic NC16A domain of BP180 [13]. Moreover, it was proved that BP patients with ND disease have anti-BP230 serum autoantibodies that bind both epithelial (BPAG1e) and neuronal (BPAG1a) isoforms [14]. Cases of psoriasis preceding autoimmune blistering dermatoses, particularly BP, were reported in the literature, including a report from our university department [15]. We have recently seen a male octogenarian with psoriasis lasting many decades that underwent erythrodermic exacerbation with extensive scaling 2 weeks before presentation followed by development of numerous intensely itchy blisters especially on palms and soles just a week before presentation. On the ground of clinical picture and results of laboratory tests, dyshidrotic BP coexistent with erythrodermic psoriasis was diagnosed. Therefore, conceivably, progressing conformational modifications, in numerous combinations, of neuronal, epidermal or both neuronal and epidermal isoforms of BP antigens triggered by a plethora of stimuli may induce the development of BP-type autoimmunity and finally clinically overt BP.

The objective of this study was to analyze the prevalence of ND in BP patients in a setting of Central European dermatology department.

\section{Materials and methods}

The medical history of $94 \mathrm{BP}$ patients diagnosed at molecular level with direct immunofluorescence, indirect immunofluorescence mosaic (IIFm) (Euroimmun, Germany) and ELISA (Euroimmun, Germany) [26 BP patients with ND (BP+ND) and $68 \mathrm{BP}$ patients without ND (BP$\mathrm{ND})$ ] hospitalized in Central European university dermatology department between December 2006 and May 2015 (BP) were reviewed for the presence of ND records. ND included: Parkinson's disease, dementia, stroke, hear loss, tinnitus, blindness, vertigo, neurosyphilis, systemic sclerosis, and epilepsy. The study group is described in the Tables 1 and 2.

Table 1 Studied groups

\begin{tabular}{ll}
\hline & BP $(n=94)$ \\
\hline M/F ratio & 0.59 \\
Mean age \pm SD & $75 \pm 11$ \\
Patients with more than one ND & 3 \\
\hline
\end{tabular}

$B P$ bullous pemphigoid; $n$ number of cases; $M / F$ ratio male to female ratio; $N D$ neurodegenerative disease; $S D$ standard deviation
Table 2 Neurodegenerative diseases in studied groups

\begin{tabular}{lc}
\hline ND & BP $(n=94)$ \\
\hline Stroke & 12 \\
Dementia & 5 \\
Parkinson's disease & 5 \\
Hear loss & 1 \\
Vertigo & 2 \\
Blindness & 1 \\
Tinnitus & 1 \\
Systemic sclerosis & 1 \\
Epilepsy & 1 \\
Neurosyphilis & 1 \\
\hline
\end{tabular}

$B P$ bullous pemphigoid; $n$ number of cases; $N D$ neurodegenerative disease

\section{Results}

The prevalence of ND in the analyzed group was $27.66 \%$, stroke- $12.77 \%$, Parkinson's disease- $5.32 \%$, dementia- $5.32 \%$ and number of patients with more than one ND reached $3.19 \%$.

\section{Discussion}

$\mathrm{BP}$ is the most frequent autoimmune blistering dermatosis (incidence 7-43 per million population per year), debilitating condition affecting mostly the elderly [16, 17]. Recently re-estimated risk of death in BP patients seems to be more than two- to sixfold higher than in general population $[18,19]$, while the 1-year survival is reported to be $62 \%$ [19]. Because of population ageing, BP and associated disorders become burning socioeconomic problem $[18,19]$. Thus, there is an urgent need for in-depth studies concerning disease mechanisms. The course of BP in patients with anti-BP180 IgG, and thus ND rate, may differ from that seen in individuals with solely anti-BP230 IgG (pemphigoid anti-BP230) or with coexisting anti-BP230 and anti-BP180 IgG [20]. In our issue-probing retrospective immunopathologic study, the occurrence of ND in BP has not been related to levels of serum IgG antibodies to either BP180 or BP230 [21]; nevertheless, detailed assessment of cerebrospinal fluid antibodies to BP180 [22] and BP230 could be more productive in that respect. The knowledge about the pathogenetic mechanisms interlinking $\mathrm{ND}$ and BP, as well as immunologic features in these patients remains scant; yet several hypotheses were coiled to elucidate this phenomenon. It was speculated that ND patients received drugs (myorelaxants, neuroleptics, aldosterone antagonists) that are known triggers of BP, yet no correlation was reported between the intake time and 
clinical onset of BP [8]. Other speculations concerned the possibility of dermal-epidermal junction (DEJ) destruction with subsequent antigen exposition and immunomodulation due to the development of decubitus ulcers in bedridden ND patients, role of stress and finally age-related immunologic dysfunction resulting in autoimmunization as both neuronal tissue and skin derive from neural crest [21]. Thus, immunosenescence may be the link between examined disorders. Remodeling of innate immunity and clonotypical immunity (significant changes in the function of $\mathrm{T}$ cells) as well as occurrence of chronic inflammatory process may promote tissue degeneration in BP and ND [23]. The genetic alteration of BPAG1a on mouse model resulted in the accumulation of intermediate filaments in motor neurons prior to neurological degeneration and dystonia. This intermediate filaments' accumulation was hypothesized to produce loss of tolerance to BPAG1a and giving cross-reactiveness with the epidermal BPAG1e [24].

The incidence of ND in our BP patients (27.66\%) seems to be in the middle part of dispersion reported in recent studies from United Kingdom, France, Czech Republic and Brazil: 22-46\% [9, 12, 17, 24, 25]. The differences between studies may derive from genetic and geographical factors. There is also a possibility that some BP-ND patients remain underdiagnosed, as the awareness about the linkage might be still low among dermatologists, who may marginalize non-dermatological conditions or simply neglect them in medical records. The incidence of cerebrovascular incident in BP patients $(12.77 \%)$ was similar to the one noted by the French group (15\%), but lower than in the United Kingdom (44.4\%) $[12,17,24]$. It is possible, that apart from genetic susceptibility, dietary habits and different living conditions (including post-war period) and high death rate observed in stroke patients in Poland might play a role in the number of reported cerebrovascular incidents. Parkinson's disease was present in $5.32 \%$ of our BP patients in comparison to $9 \%$ in France and $30 \%$ in United Kingdom [12, 17, 24]. Dementia rate in the studied BP group reached $5.32 \%$. A report on populational study in Midwestern Poland estimated that in people $>65$ years dementia was observed in $5.7 \%$ [26] in what seems to be in the lower part of dispersion of the epidemiologic data in this geographic region (in contrast to $13 \%$ in United Kingdom) [12] and corresponds with our findings. The low rate is speculated to be caused by underdiagnosing of dementia [27]. The study by Langan et al. reported threefold risk of developing BP in patients with Parkinson's disease and dementia, whereas twofold risk in patients with stroke and epilepsy, comparing to healthy population [9].

The pathogenetic ND-related mechanisms in BP have not been elucidated yet; yet they possibly involve autoantigens common in central nervous system and epidermis. We have recently seen a middle-aged female, much younger than stereotypical octogenarian with BP, with coexistent BP
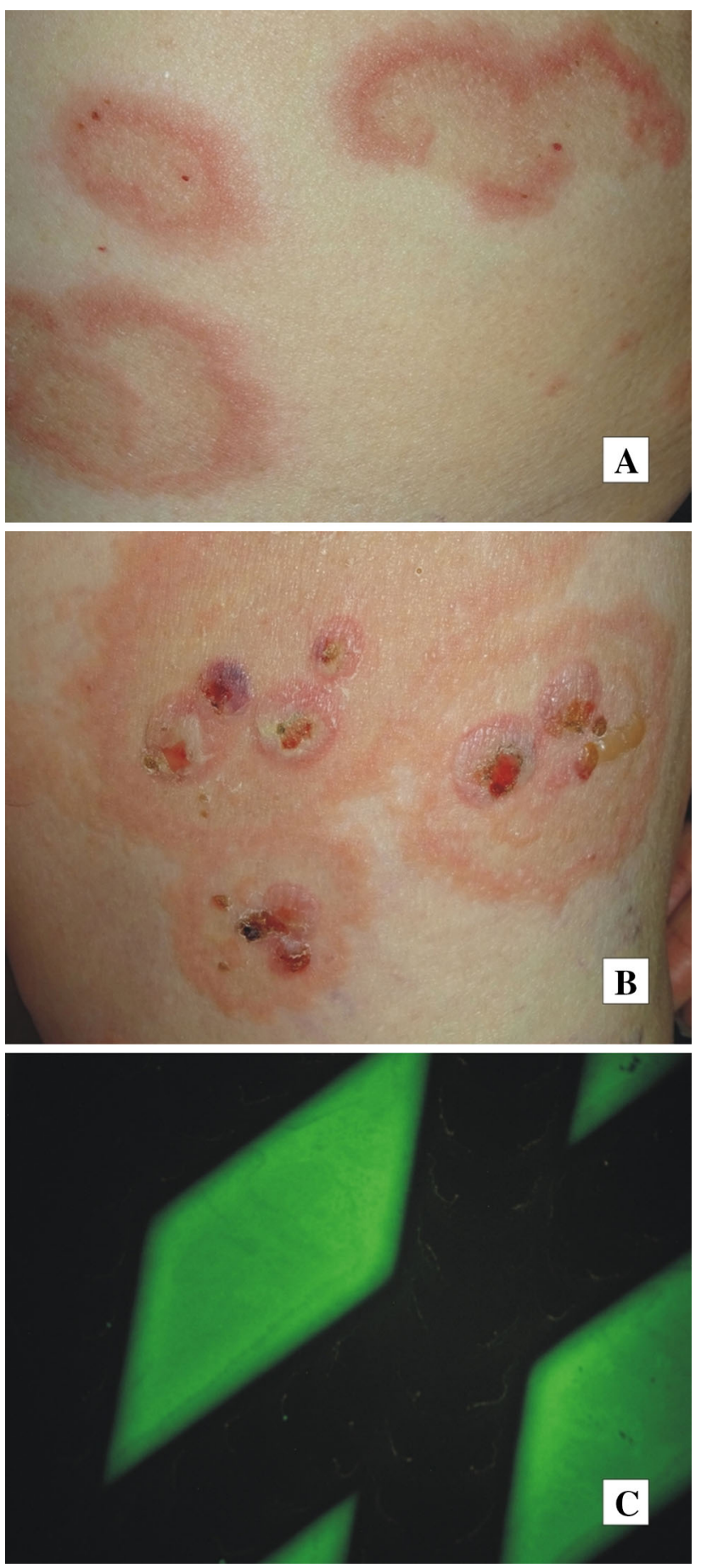

Fig. 1 Bullous pemphigoid coexistent with late syphilis. Erythema annulare centrifugum (deep variety)-like eruption on the trunk (a). Ruptured blisters on erythematous base affecting medial surface of a thigh (b). Indirect immunofluorescence mosaic: serum IgG autoantibodies against the tetrameric BP180 NC16A spots (antigen dots) (c)

clinically resembling a deep variety of erythema annulare centrifugum (Fig. 1a, b) and marginally symptomatic late syphilis causing memorization impairment [28]. Importantly, both BP and syphilis were diagnosed at the usable 
molecular level with ELISA and IIFm for serum IgG antibodies to BP180 (Fig. 1c), and chemiluminescent microparticle immunoassay for serum antibodies to Treponema pallidum recombinant antigens (TpN15, TpN17, TpN47). Such coexistences may be yet another argument for experimentally exploring plausible BP-specific immunopathogenetic links between BP and ND.

\section{Conclusion}

Although BP is relatively rare disease, patients should be routinely screened for underlying/concomitant ND. On the other hand, patients with ND should be carefully followedup for BP development. Since population aging is conceivably responsible for rising number of $\mathrm{BP}$ cases, the plausible BP-specific immunopathogenetic relationship between $\mathrm{BP}$ and ND deserves to be further experimentally explored.

\section{Compliance with ethical standards}

Ethical approval This study is part of the project approved by the local ethics committee: No 560/15.

Conflict of interest On behalf of all authors, the corresponding author states that there is no conflict of interest.

Statement of human and animal rights All procedures performed in studies involving human participants were in accordance with the ethical standards of the 1964 Helsinki declaration and its later amendments. No studies with animals were performed.

Informed consent Informed consent was obtained from all individual participants included in the study.

Financial support and funding This study was partly funded from grant of the Polish Ministry of Science and Higher Education 0127/IPI/2015/73 and grant of the Poznan University of Medical Sciences, Poland, No. 502-14-02220350-10256. BIOCHIP IIF mosaic was obtained from EUROIMMUN (Germany) within a framework of a multicenter project: "A European prospective study on serum antibodies against target antigens of bullous autoimmune diseases and genetic susceptibility". Paweł Pietkiewicz was financially supported by Poznan University of Medical Sciences PhD Scholarship.

Open Access This article is distributed under the terms of the Creative Commons Attribution 4.0 International License (http://crea tivecommons.org/licenses/by/4.0/), which permits unrestricted use, distribution, and reproduction in any medium, provided you give appropriate credit to the original author(s) and the source, provide a link to the Creative Commons license, and indicate if changes were made.

\section{References}

1. Liu Z, Shapiro SD, Zhou X et al (2000) A critical role for neutrophil elastase in experimental bullous pemphigoid. J Clin Invest 105:113-123
2. Tambe S, Häfliger S, Borradori L (2013) Clinical challenges and recent advances in the diagnosis of bullous pemphigoid. Expert Rev Dermatol 8:407-16. http://www.medscape.com/viewarticle/ 810614. Accessed 14 July 2015

3. Schmidt E, Zillikens D (2013) Pemphigoid diseases. Lancet 381:320-332

4. Poliakova K, Adebola A, Leung CL et al (2014) BPAG1a and b associate with EB1 and EB3 and modulate vesicular transport, Golgi apparatus structure, and cell migration in C2.7 myoblasts. PLoS One 9:e107535

5. Brown A, Lemieux N, Rossant J et al (1994) Human homolog of a mouse sequence from the dystonia musculorum locus is on chromosome 6p12. Mamm Genome 5:434-437

6. Ryan SD, Ferrier A, Sato T et al (2012) Neuronal dystonin isoform 2 is a mediator of endoplasmic reticulum structure and function. Mol Biol Cell 23:553-566

7. Edvardson S, Cinnamon Y, Jalas C et al (2012) Hereditary sensory autonomic neuropathy caused by a mutation in dystonin. Ann Neurol 71:569-572

8. Stinco G, Codutti R, Scarbolo M et al (2005) A retrospective epidemiological study on the association of bullous pemphigoid and neurological diseases. Acta Derm Venereol 85:136-139

9. Langan SM, Groves RW, West J (2011) The relationship between neurological disease and bullous pemphigoid: a population-based case-control study. J Invest Dermatol 131:631-636

10. Teixeira VB, Cabral R, Brites MM et al (2014) Bullous pemphigoid and comorbidities: a case-control study in Portuguese patients. An Bras Dermatol 89:274-279

11. Brick KE, Weaver CH, Savica R et al (2014) A population-based study of the association between bullous pemphigoid and neurologic disorders. J Am Acad Dermatol 71:1191-1197

12. Taghipour K, Chi C-C, Vincent A et al (2010) The association of bullous pemphigoid with cerebrovascular disease and dementia: a case-control study. Arch Dermatol 146:1251-1254

13. Messingham K, Narayanan N, Aust S et al (2015) Collagen XVII autoantibodies are present in Parkinson's disease patients and colocalize with tyrosine hydroxylase in the substantia nigra. J Invest Dermatol 132:S14

14. Chen J, Li L, Chen J et al (2011) Sera of elderly bullous pemphigoid patients with associated neurological diseases recognize bullous pemphigoid antigens in the human brain. Gerontology 57:211-216

15. Dmochowski M, Neumann E, Bowszyc-Dmochowska M (1993) A case with coexistent bullous pemphigoid and psoriasis vulgaris. Immunofluorescence and immunoblot study. Post Dermatol 10:19-27

16. Lo Schiavo A, Ruocco E, Brancaccio G et al (2013) Bullous pemphigoid: etiology, pathogenesis, and inducing factors: facts and controversies. Clin Dermatol 31:391-399

17. Tarazona MJM, de Mota ANCM, Gripp AC et al (2015) Bullous pemphigoid and neurological disease: statistics from a dermatology service. An Bras Dermatol 90:280-282

18. Langan SM, Smeeth L, Hubbard R et al (2008) Bullous pemphigoid and pemphigus vulgaris-incidence and mortality in the UK: population based cohort study. BMJ 337:a180

19. Joly P, Baricault S, Sparsa A et al (2012) Incidence and mortality of bullous pemphigoid in France. $\mathrm{J}$ Invest Dermatol 132:1998-2004

20. Dmochowski M, Gornowicz-Porowska J, Pietkiewicz $\mathrm{P}$ et al (2013) Are patients with anti-BP230 IgG antibodies but without anti-BP180NC16a IgG antibodies suffering from less stereotypical varieties of bullous pemphigoid? International Pre IID Satellite Symposium on Autoimmune Bullous Diseases; Lübeck, Germany, 6-7th May 2013

21. Gornowicz-Porowska J, Pietkiewicz P, Bowszyc-Dmochowska $M$ et al (2015) Schorzenia neurodegeneracyjne a pemfigoid 
pęcherzowy-retrospektywne badanie immunopatologiczne. Przegl Dermatol 102:89-90

22. Chen J, Duan Y, Chen L et al (2014) Anti-bullous pemphigoid autoantigen BP180 antibodies exist in sera and cerebrospinal fluid samples of patients with stroke and dementia. J Invest Dermatol 134:S26

23. Noe MH, Messingham K, Aust S et al (2015) Bullous pemphigoid patients exhibit increased markers of immunosenescence. 2015 Annual Meeting of the SID; Atlanta, GA, USA, 6-9th May 2015, Meeting Program, S1:LB738

24. Cordel N, Chosidow O, Hellot M-F et al (2007) French Study Group of Bullous Diseases. Neurological disorders in patients with bullous pemphigoid. Dermatol Basel Switz 215:187-191

25. Jedlickova H, Hlubinka M, Pavlik T et al (2010) Bullous pemphigoid and internal diseases-a case-control study. Eur J Dermatol 20:96-101
26. Rossa G (1997) The prevalence of Alzheimer's type dementia and vascular dementia in the district of Swiebodzin. Psychiatr Pol 31:121-134

27. Wender M, Mularczyk J, Modestowicz R (1989) Epidemiology of Alzheimer's disease in the selected region of Wielkopolska (town and commune Steszew). Przegl Epidemiol 44:215-221

28. Gornowicz-Porowska J, Bartkiewicz P, Pietkiewicz P et al (2015) Pemfigoid pęcherzowy $\mathrm{z}$ wykwitami typu rumienia obrączkowatego odśrodkowego w odmianie głębokiej u kobiety w średnim wieku w przebiegu zakażenia Treponema pallidum. Przegl Dermatol 102:149-150 